\title{
Coumestrol Down-Regulates Melanin Production in Melan-a Murine Melanocytes through Degradation of Tyrosinase
}

\author{
Jeong Ah Hwang, Nok Hyun Park, Yong Joo Na, Hae Kwang Lee, John Hwan Lee, \\ Yong Jin Kim, and Chang Seok Lee* \\ Amorepacific R\&D Corporation Center; 1920 Yonggu-daero, Giheung-gu, Yongin-si, Gyeonggi-do 17074, Korea. \\ Received October 25, 2016; accepted January 18, 2017
}

\begin{abstract}
Pigmentation reflects skin darkening caused by melanin production, but excessive melanin synthesis may cause problems, such as melasma, solar lentigo, dark spots, and freckles. Considerable effort has been devoted to alleviating these undesired symptoms through the development of safe and effective depigmenting agents. Coumestrol, a plant-derived natural isoflavone with an estrogen-like structure and actions, is known to have anti-aging ability, but its potential depigmenting efficacy has not been evaluated. In the present study, we investigated the effects of coumestrol on melanin synthesis in normal melan-a murine melanocytes. Coumestrol significantly reduced melanin synthesis in a concentration-dependent manner up to a concentration of $25 \mu \mathrm{M}$ without causing cytotoxicity. It also brightened tissue in an artificial skin model (MelanoDerm) that incorporates both human keratinocytes and melanocytes. Interestingly, although coumestrol did not inhibit tyrosinase activity or transcript level in melan-a cells, it clearly decreased the expression level of tyrosinase protein at a concentration of $25 \mu \mathrm{M}$. This coumestrol-induced reduction in tyrosinase protein levels was prevented by pretreatment with the proteasome inhibitor MG-132 or the lysosomal proteolysis inhibitor chloroquine. Collectively, our findings indicate that coumestrol exerts an inhibitory effect on melanin synthesis in melan-a cells, at least in part, through degradation of tyrosinase. These findings suggest that coumestrol is a good candidate for use in depigmentary reagents from a cosmetic and clinical perspective.
\end{abstract}

Key words coumestrol; phytoestrogen; melanogenesis; tyrosinase

Melanin is a key determinant of skin and hair color. Melanin absorbs UV light and protects the skin from the damaging effects of UV light and UV-generated free radicals. ${ }^{1)}$ Despite the beneficial role of melanin in the skin, excessive production and accumulation of melanin results in several skin disorders, including acquired hyperpigmentation syndromes such as melasma, age spots and freckles, among others. ${ }^{2)}$

Tyrosinase, a potential melanogenic protein, is the rate-limiting enzyme in melanogenesis. It is translated and processed in the endoplasmic reticulum (ER) and Golgi apparatus, and then transported to melanosomes, where melanin is synthesized and accumulates. ${ }^{3)}$ Tyrosinase can be degraded by two pathways: ER-associated protein degradation (ERAD) through the ubiquitin proteasome system, ${ }^{4)}$ and endosome/lysosomemediated degradation. ${ }^{5)}$ Because tyrosinase is a potent inducer of melanin synthesis, tyrosinase inhibitors could be effective skin-whitening agents. ${ }^{6)}$

Coumestrol, a phytoestrogen, is a soy-derived natural compound that can bind to the estrogen receptor. ${ }^{7)}$ Phytoestrogens exert many biological effects, as exemplified by the inhibitory effect of genistein, daidzein, and coumestrol on tumor necrosis factor (TNF)-induced bone resorption in RAW264.7 cells. ${ }^{8)}$ Coumestrol can also bind to the surface of the pregnane $\mathrm{X}$ receptor (PXR) and antagonize PXR activities in primary hepatocytes. ${ }^{9)}$ In addition, coumestrol shows anti-aging effects on skin and inhibits UVB-induced matrix metallopeptidase (MMP)-1 expression by suppressing the kinase activity of fms-related tyrosine kinase 3 (FLT3) ${ }^{10)}$ However, no studies have investigated the effects of coumestrol on melanogenesis or skin hyperpigmentation. In this study, we provide the first report of the anti-melanogenic actions of coumestrol, demonstrating that the effects of coumestrol are mediated by down-regulation of the protein level of tyrosinase, a key melanogenic enzyme.

\section{MATERIALS AND METHODS}

Materials Coumestrol was obtained from Indofine (Hillsborough, NJ, U.S.A.). Arbutin, kojic acid, tyrosine, mushroom tyrosinase, cycloheximide, MG-132, and chloroquine were purchased from Sigma (St. Louis, MO, U.S.A.). The antibody against microphthalmia-associated transcription factor (MITF) was purchased from Proteintech (Rosemont, IL, U.S.A.). Antibodies against tyrosinase and tyrosinase-related protein-1 (Tyrp-1) and -2 (Tyrp-2) were a kind gift from Dr. V. J. Hearing (National Institutes of Health, Bethesda, MD, U.S.A.).

Cell Culture Melan-a cells, an immortalized mouse melanocyte cell line derived from C57BL6 mice, were maintained as monolayers at $37^{\circ} \mathrm{C}$ in a humidified $10 \% \mathrm{CO}_{2}$ incubator in RPMI-1640 medium (Lonza, Basel, Switzerland) containing $10 \%$ fetal bovine serum (FBS; Grand Island, Gibco, NY, U.S.A.), $100 \mathrm{U} / \mathrm{mL}$ potassium penicillin and $100 \mathrm{mg} / \mathrm{mL}$ streptomycin sulfate (PS; Lonza), and phorbol 12-myristate 13-acetate (PMA; Sigma-Aldrich).

Cell Viability Assay Melan-a cells were seeded onto 96-well plates $\left(1 \times 10^{4}\right.$ cells/well) and cultured with coumestrol for $72 \mathrm{~h}$, after which an 3-[4,5-dimethylthiazol-2-yl]-2,5-diphenyltetrazolium bromide (MTT) solution was added and cells were incubated for $30 \mathrm{~min}$. The dark blue formazan crystals formed in intact cells were dissolved in dimethylsulfoxide (DMSO), and absorbance was measured at $570 \mathrm{~nm}$ using a microplate reader.

Melanin Assay Melan-a cells were seeded onto 48-well plates $\left(1.5 \times 10^{4}\right.$ cells/well $)$ and treated the next day with the 
indicated concentrations of coumestrol. After $3 \mathrm{~d}$, media were replaced with fresh media containing coumestrol, and cells were incubated for an additional $3 \mathrm{~d}$. Media were then removed, and the cells were washed with Dulbecco's phosphate-buffered saline (DPBS). Thereafter, $100 \mu \mathrm{L}$ of $2 \mathrm{~N}$ $\mathrm{NaOH}$ containing $10 \%$ DMSO was added to each well and plates were incubated for $30 \mathrm{~min}$ at $60^{\circ} \mathrm{C}$ to cause cell lyses. Cell lysates were transferred to a 96 -well plate and absorbance was measured at $475 \mathrm{~nm}$. The total protein content in each experimental group was then measured using a DC Protein Assay (BioRad, Hercules, CA, U.S.A.). After normalizing the calculated melanin contents to total protein contents, melanin contents per protein of each experimental group are obtained. Independent experiments were carried out three times.

Mushroom Tyrosinase Activity Assay A cell-free assay system was used to investigate the direct effects of the indicated concentrations of coumestrol on tyrosinase activity. Briefly, $100 \mu \mathrm{L}$ of phosphate buffer containing coumestrol was mixed with 10 units of mushroom tyrosinase, after which $50 \mu \mathrm{L}$ of $0.03 \%$ tyrosine in distilled water was added. After incubation at $37^{\circ} \mathrm{C}$ for $10 \mathrm{~min}$, absorbance was measured at $475 \mathrm{~nm}$. Independent experiments were carried out three times.

Skin Equivalent (MelanoDerm) Assay African-American human epidermal equivalent (MelanoDerm; MatTek Corp., Ashland, MA, U.S.A.) was grown at $37^{\circ} \mathrm{C}$ in a humidified $5 \% \mathrm{CO}_{2}$ incubator, and the media were replaced every $2 \mathrm{~d}$. Skin equivalent was treated with different concentrations of coumestrol, diluted in DPBS; $1 \%$ kojic acid was used as a positive control. Pigmentation level in skin equivalent was calculated by comparing variations in $L^{*}$ values (a lightness/ darkness index) on day 0 and 9 , and calculating the difference between them $(\Delta L)$.

RNA Isolation and Quantitative (q)RT-PCR Melan-a cells cultured with different concentrations of coumestrol for $8 \mathrm{~h}$ were lysed, and total RNA was extracted using an RNeasy mini kit (Qiagen, CA, U.S.A.) following the manufacturer's instructions. cDNA was synthesized from total RNA using a Superscript Reverse Transcriptase (RT) II kit (Invitrogen, Carlsbad, CA, U.S.A.) by incubating at $50^{\circ} \mathrm{C}$ for $1 \mathrm{~h}$ and then denaturing at $95^{\circ} \mathrm{C}$ for $5 \mathrm{~min}$. The quantitative real time-PCR (qRT-PCR) experiments were performed on an Applied Biosystems 7500 Fast Real-time PCR System (Applied Biosystems, Foster City, CA, U.S.A.) using TaqMan Gene Expression Assay primer sets (Applied Biosystems).

Western Blotting Melan-a cells were seeded in 6-well plates and treated with coumestrol for $72 \mathrm{~h}$. Cells were then lysed by incubation with RIPA lysis buffer for $20 \mathrm{~min}$ after which $10 \mu \mathrm{g}$ of lysate protein was separated by sodium dodecyl sulfate-polyacrylamide gel electrophoresis (SDS-PAGE) on $10 \%$ gels. Proteins were transferred to nitrocellulose membranes (Invitrogen, CA, U.S.A.), which were then incubated with blocking solution (5\% skim milk) for $2 \mathrm{~h}$ at room temperature, followed by incubation with the specific primary antibody at $4^{\circ} \mathrm{C}$ overnight. The membranes were washed with Tris-buffered saline containing 0.1\% Tween-20 (TBST) and then incubated with horseradish peroxidase (HRP)-conjugated secondary antibodies (Santa Cruz Biotechnology, CA, U.S.A.) for $1 \mathrm{~h}$ at room temperature. After washing, immunoreactive proteins were detected by enhanced chemiluminescence (ECL) using ECL Prime Western blotting Detection Reagent (GE Healthcare, U.K.). For quantitative analysis, band den- sities were measured by scanning densitometry using the ChemiDoc XRS+ system (Bio-Rad, CA, U.S.A.).

Statistical Analysis Data are expressed as means + standard deviation (S.D.) values, and Student's $t$-test was used for statistical comparisons. A $p$-value $<0.05$ was considered statistically significant (individual $p$-values are given in figure legends). For real-time PCR data, differences in the amount of target between samples, normalized to endogenous glyceraldehyde-3-phosphate dehydrogenase (GAPDH), were determined using the $2^{-\Delta \Delta \mathrm{C}_{\mathrm{T}}}$ method.

\section{RESULTS}

Before evaluating coumestrol (Fig. 1A) activities on melana cells, we measured cellular toxicity to determine an appropriate coumestrol concentration. Melan-a cells were cultured for $3 \mathrm{~d}$ with the indicated concentrations of coumestrol, and their viability was assessed by MTT assay (Fig. 1B). Melan-a cell viability trended lower following treatment with coumestrol at concentrations up to $25 \mu \mathrm{M}$ compared with controls, and was overtly cytotoxic at concentrations of $50 \mu \mathrm{M}$ or more. Therefore, subsequent experiments using melan-a cells were conducted using $25 \mu \mathrm{M}$ coumestrol or less.

Next, melan-a cells were treated with the indicated concentrations of coumestrol, and total melanin was measured $6 \mathrm{~d}$ later. A quantitative analysis showed that coumestrol treatment significantly decreased melanin content in melan-a cells at all concentrations tested (Fig. 1C). The whitening ability of coumestrol was also investigated in human skin equivalent using the MelanoDerm preparation as an epidermis model. Treatment of MelanoDerm, consisting of well-differentiated human keratinocytes and melanocytes, with different concentrations of coumestrol for $9 \mathrm{~d}$ revealed no evidence of cytotoxicity. As shown in Fig. 1D, coumestrol brightened the color of the skin tissue at all concentrations tested. An analysis of the results showed that coumestrol increased the $\Delta L$ value of skin equivalent in a concentration-dependent manner compared with controls (Fig. 1E). Collectively, these results indicate that coumestrol decreases melanin content in murine and human melanocytes.

To define the anti-melanogenic mechanism of coumestrol, we focused on the activation and expression of tyrosinase, a key enzyme in melanin production and an inhibitory target of many studies designed to develop potent skin-depigmenting ingredients. Accordingly, we first tested the ability of coumestrol to inhibit the tyrosine hydroxylase activity of mushroom tyrosinase using a cell-free, enzyme-substrate reaction assay in which coumestrol could be applied at higher concentrations. As shown in Fig. 2A, coumestrol did not inhibit mushroom tyrosinase activity at concentrations up to $50 \mu \mathrm{M}$, suggesting that coumestrol acts through a mechanism distinct from direct inhibition of tyrosinase activity to decrease melanogenesis in melan-a cells. To test this possibility, we treated melan-a cells with coumestrol and then evaluated the levels of mRNA for the melanogenic proteins, Tyrp-1 and Tyrp-2, as well as tyrosine. Unexpectedly, we found that coumestrol did not inhibit transcription of melanogenic genes (Fig. 2B). However, Western blot analyses revealed that coumestrol did decrease tyrosinase protein levels, albeit only at a concentration of $25 \mu \mathrm{M}$; by contrast, coumestrol had no effect on Tyrp-1, Tyrp-2 or MITF protein levels (Fig. 2C). Collectively, these findings suggest 
A<smiles>O=c1oc2cc(O)ccc2c2oc3cc(O)ccc3c12</smiles>

C

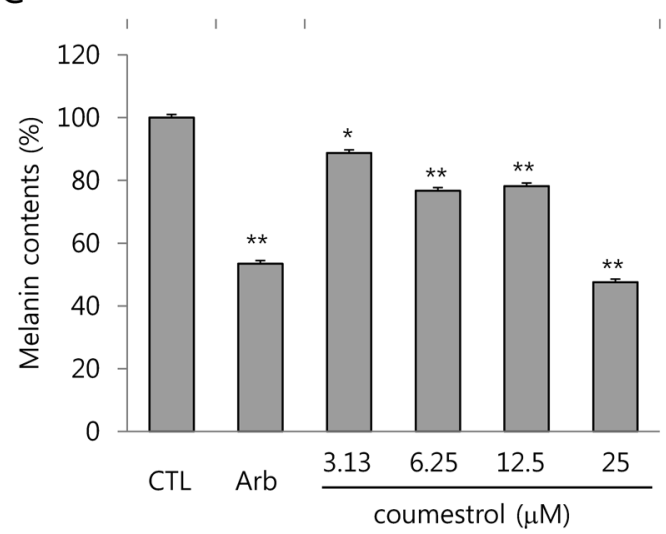

E

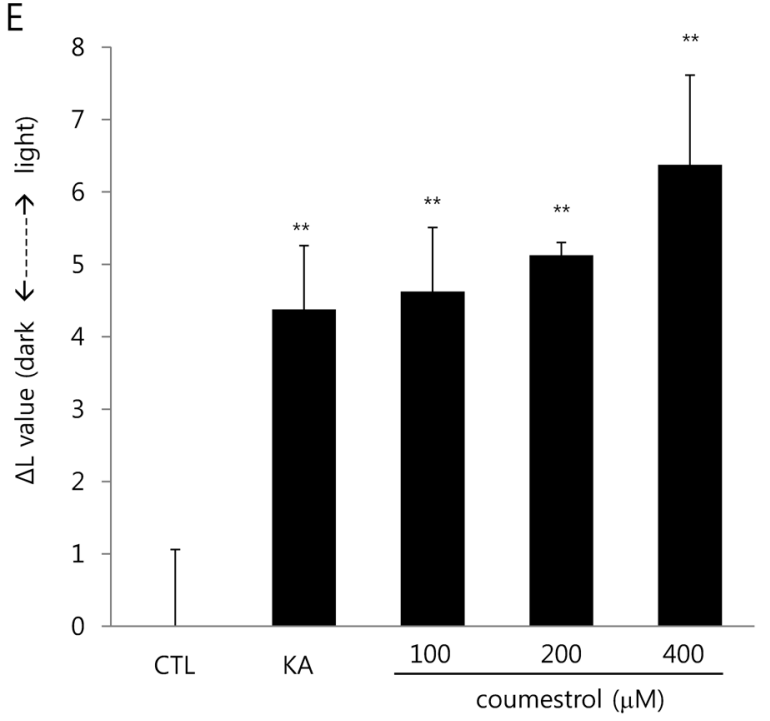

B

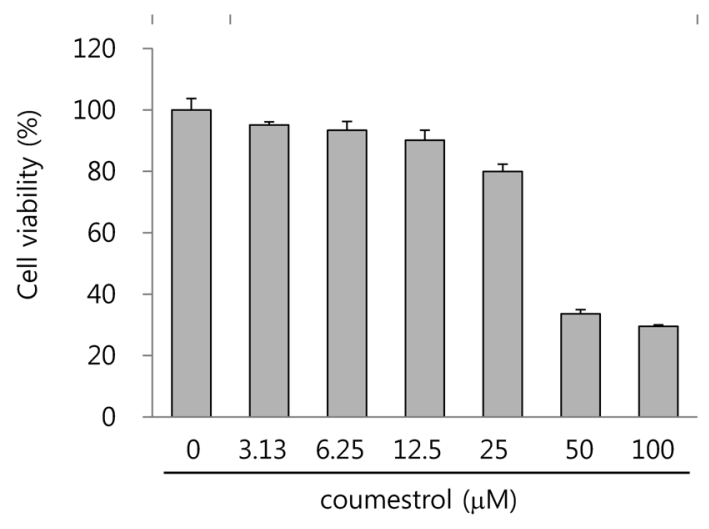

D

Day 0

Day 9

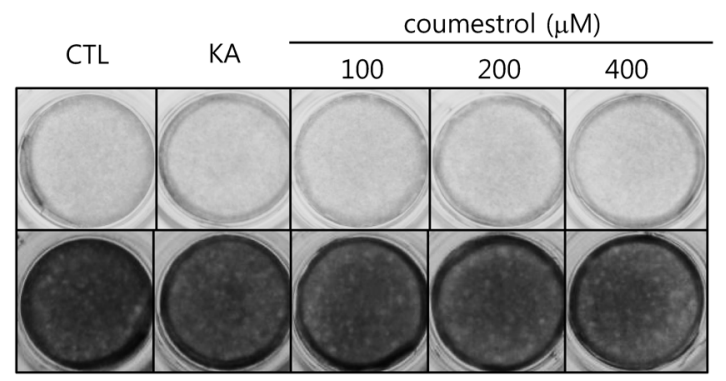

Fig. 1. The Depigmentation Efficacy of Coumestrol in Melan-a Cells and Human Skin Equivalent

(A) Chemical structure of coumestrol. (B) Effects of coumestrol on the viability of melan-a cells. Cells were treated with the indicated concentrations of coumestrol for $72 \mathrm{~h}$. Cell viability was determined by MTT assay, as described in Materials and Methods. Values are means \pm S.D. of three determinations, expressed as a percentage of controls. (C) Effects of coumestrol on melanin synthesis in melan-a cells. Melan-a cells were treated with the indicated concentrations of coumestrol or arbutin (Arb; $200 \mu \mathrm{M})$, used as a positive control, for $3 \mathrm{~d}$, and melanin content was measured $6 \mathrm{~d}$ later. Values are means \pm S.D. of three determinations expressed as a percentage of controls. (D, E) Effects of coumestrol on human skin equivalent. Human skin equivalent (MelanoDerm; $n=3$ ) was treated with the indicated concentrations of coumestrol or kojic acid (1\%) for $9 \mathrm{~d}$. After treatment, skin equivalent was photographed (D) and $\Delta L$ value (degree of lightness compared with vehicle-treated controls) for each test sample was calculated using the following formula: $\Delta L$ value $=($ the change in $L$ value for vehicle-treated control) $-($ the change in $L$ value for coumestrol-treated sample) (E). $\left({ }^{*} p<0.05,{ }^{* *} p<0.01\right.$ vs. control).

that the anti-melanogenic effect of coumestrol is mediated, at least in part, by regulation of tyrosinase at the translational or post-translational level, and not at the transcriptional level.

Tyrosinase is degraded endogenously by proteasomesmulticatalytic proteinase complexes that selectively degrade intracellular ubiquitinated proteins. To determine whether proteasomal or lysosomal pathways are involved in mediating tyrosinase degradation by coumestrol, we evaluated tyrosinase protein levels in melan-a cells treated with MG-132, a proteasome inhibitor, and/or chloroquine, a lysosomal proteolysis inhibitor. In these experiments, melan-a cells were serumstarved for $24 \mathrm{~h}$, then treated with cycloheximide to inhibit protein synthesis. MG-132 and/or chloroquine were added, and cells were incubated for $1 \mathrm{~h}$ before incubating with coumestrol for $6 \mathrm{~h}$. After treatment, tyrosinase expression levels were assessed by Western blotting. As shown in Fig. 2D, coumestrolinduced decreases in tyrosinase protein levels were attenuated by pretreatment with MG-132 and chloroquine, indicating that coumestrol suppresses tyrosinase protein levels by promoting proteasomal- and lysosomal-mediated degradation of tyrosinase. 

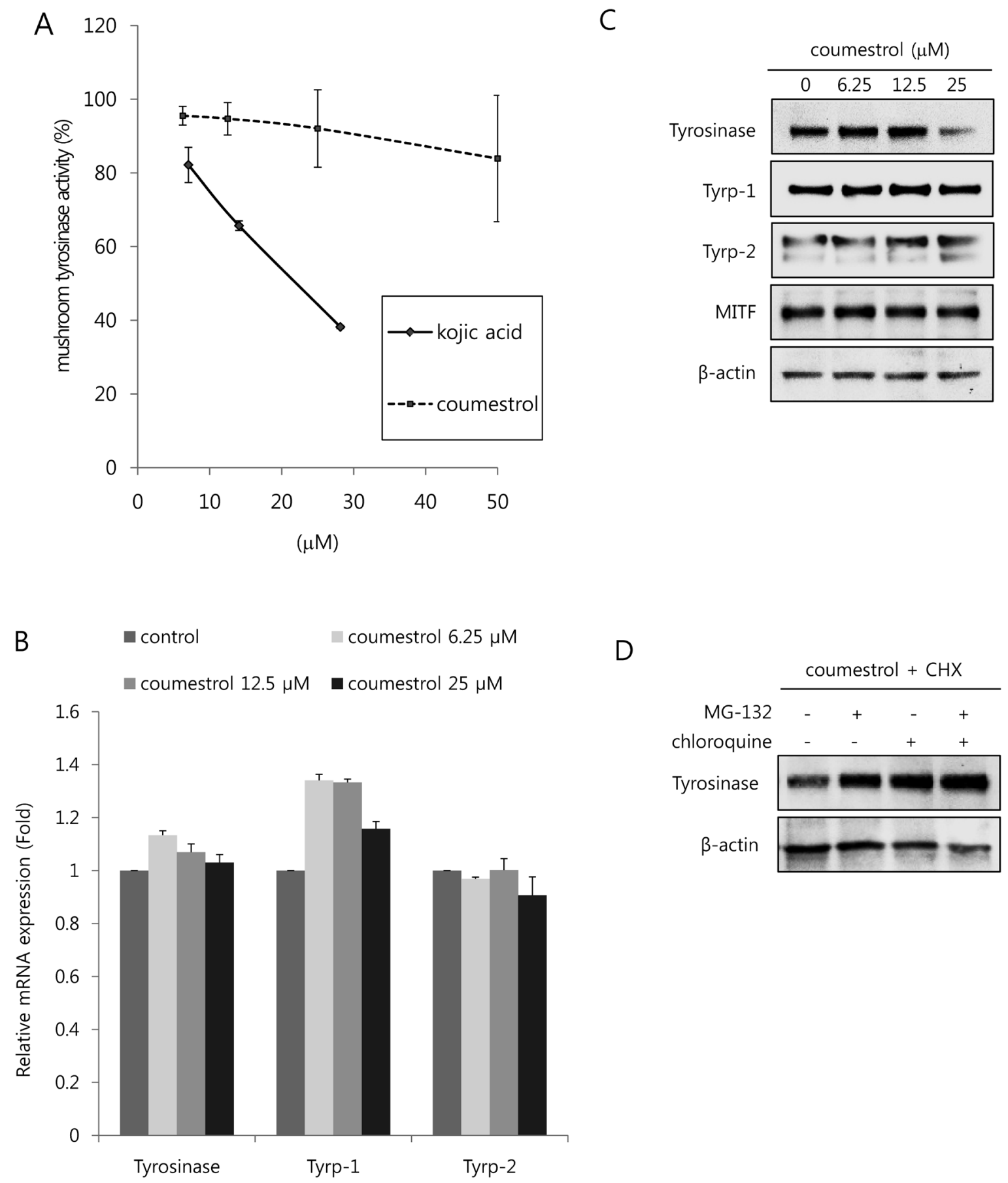

Fig. 2. The Mechanism Underlying the Anti-melanogenic Activity of Coumestrol

(A) Effects of coumestrol on tyrosinase activity in cell-free assays. The indicated concentrations of coumestrol and kojic acid were incubated with mushroom tyrosinase. Values are means \pm S.D. of three determinations, expressed as a percentage of controls. (B) Effects of coumestrol on mRNA expression levels of melanogenesis-related genes in melan-a cells. Cells were treated with coumestrol for $8 \mathrm{~h} . \mathrm{mRNA}$ levels were analyzed by qRT-PCR using GAPDH as an internal standard. mRNA/GAPDH ratios of melanogenesis-related gene are expressed relative to those in controls. Values are means \pm S.D. of three determinations. (C) Effects of coumestrol on the levels of melanogenesis-related proteins in melan-a cells. Melan-a cells were incubated with the indicated concentrations of coumestrol. After $72 \mathrm{~h}$, the cells were washed with DPBS and harvested. Protein levels of tyrosinase, Tyrp-1, Tyrp-2, MITF and $\beta$-actin were determined by Western blotting, as described in Materials and Methods. Values are means \pm S.D. of three determinations. (D) Effects of coumestrol on tyrosinase degradation in melan-a cells. Cells were pretreated first with cycloheximide ( $70 \mu \mathrm{M})$ and then with MG-132 $(25 \mu \mathrm{M})$ and/or chloroquine $(100 \mu \mathrm{M})$, followed by treatment with $25 \mu \mathrm{m}$ coumestrol. After treatment, tyrosinase levels were examined by Western blot analysis. $\beta$-Actin was used as internal control for each condition.

\section{DISCUSSION}

Previous studies have reported various effects of coumestrol, showing for example that coumestrol suppresses hypoxiainducible factor- $1 \alpha$ by inhibiting reactive oxygen species (ROS)-mediated sphingosine kinase 1 activity, ${ }^{11)}$ and induces mitochondrial biogenesis by activating Sirt1. ${ }^{12)}$ However, studies of the efficacy of coumestrol on skin are limited. In the current study, we provide the first demonstration of the antimelanogenic effects of coumestrol. In Fig. 1C, we showed that coumestrol exerts concentration-dependent anti-melanogenesis effects in melan-a cells at $3.13,6.25,12.5$ and $25 \mu \mathrm{M}$, concen- 
trations that caused no significant cytotoxicity. The skin-whitening effect of coumestrol was also assessed in human skin equivalent, where higher concentrations could be tested. These experiments revealed that $100 \mu \mathrm{M}$ coumestrol was as effective as $1 \%$ kojic acid $(70.37 \mathrm{~mm})$, indicating that coumestrol would be more effective for skin whitening than this well-known whitening agent. ${ }^{13)}$

To identify the molecular target of coumestrol, we carried out qRT-PCR assays and performed Western blotting in melan-a cells. The results of these analyses showed that coumestrol-induced inhibition of melanin synthesis did not result from a decrease in the transcription of genes encoding melanogenic proteins (tyrosinase, Tyrp-1 or Tyrp-2). Instead, coumestrol suppressed tyrosinase protein levels, but only at a concentration of $25 \mu \mathrm{M}$. These results suggest that coumestrol at $25 \mu \mathrm{M}$ degraded tyrosinase in melan-a cells. To determine whether proteasomal or lysosomal pathways are involved in mediating coumestrol-induced tyrosinase degradation, we conducted an analysis using MG-132, a proteasome inhibitor, and chloroquine, a lysosomal proteolysis inhibitor. These analyses showed that coumestrol-induced decreases in tyrosinase protein levels were attenuated by pretreatment with MG-132 and/or chloroquine, demonstrating that coumestrol exerts its anti-melanogenesis effect through proteasomal- and lysosomal-mediated tyrosinase degradation. However, we found that coumestrol induced tyrosinase degradation only at $25 \mu \mathrm{M}$, although lower concentrations $(3.13,6.25$ and $12.5 \mu \mathrm{M})$ of coumestrol reduced melanin content in melan-a cells. Thus, we conclude that coumestrol exerts an inhibitory effect on melanin synthesis in part through tyrosinase degradation, and that other concurrent mechanisms are also involved in coumestrol-induced depigmentation. Further studies of the relationship between coumestrol and tyrosinase ubiquitination are needed to confirm the details of this mechanism.

Collectively, our investigation of the whitening effect of coumestrol using multiple evaluation systems, including human skin equivalent, demonstrates that coumestrol could be used as a potent skin-whitening ingredient for hyperpigmentation care.

Acknowledgment This research was supported and funded by the Application Technology Research Institute of the AmorePacific R\&D Center.

Conflict of Interest The authors declare no conflict of interest.

\section{REFERENCES}

1) Swalwell H, Latimer J, Haywood RM, Birch-Machin MA. Investigating the role of melanin in UVA/UVB- and hydrogen peroxideinduced cellular and mitochondrial ROS production and mitochondrial DNA damage in human melanoma cells. Free Radic. Biol. Med., 52, 626-634 (2012).

2) Briganti S, Camera E, Picardo M. Chemical and instrumental approaches to treat hyperpigmentation. Pigment Cell Res., 16, 101-110 (2003).

3) Ando H, Ichihashi M, Hearing VJ. Role of the ubiquitin proteasome system in regulating skin pigmentation. Int. J. Mol. Sci., 10, 4428-4434 (2009).

4) Svedine S, Wang T, Halaban R, Hebert DN. Carbohydrates act as sorting determinants in ER-associated degradation of tyrosinase. $J$. Cell Sci., 117, 2937-2949 (2004)

5) Hall AM, Orlow SJ. Degradation of tyrosinase induced by phenylthiourea occurs following Golgi maturation. Pigment Cell Res., 18, 122-129 (2005)

6) Khan MT. Novel tyrosinase inhibitors from natural resources - their computational studies. Curr. Med. Chem., 19, 2262-2272 (2012).

7) Markaverich BM, Webb B, Densmore CL, Gregory RR. Effects of coumestrol on estrogen receptor function and uterine growth in ovariectomized rats. Environ. Health Perspect., 103, 574-581 (1995).

8) Karieb S, Fox SW. Phytoestrogens directly inhibit TNF- $\alpha$-induced bone resorption in RAW264.7 cells by suppressing c-fos-induced NFATc1 expression. J. Cell. Biochem., 112, 476-487 (2011).

9) Wang H, Li H, Moore LB, Johnson MD, Maglich JM, Goodwin B, Ittoop OR, Wisely B, Creech K, Parks DJ, Collins JL, Willson TM, Kalpana GV, Venkatesh M, Xie W, Cho SY, Roboz J, Redinbo M, Moore JT, Mani S. The phytoestrogen coumestrol is a naturally occurring antagonist of the human pregnane $\mathrm{X}$ receptor. Mol. Endocrinol., 22, 838-857 (2008).

10) Park G, Baek S, Kim JE, Lim TG, Lee CC, Yang H, Kang YG, Park JS, Augustin M, Mrosek M, Lee CY, Dong Z, Huber R, Lee KW. Flt3 is a target of coumestrol in protecting against UVB-induced skin photoaging. Biochem. Pharmacol., 98, 473-483 (2015).

11) Cho SY, Cho S, Park E, Kim B, Sohn EJ, Oh B, Lee EO, Lee HJ, Kim SH. Coumestrol suppresses hypoxia inducible factor $1 \alpha$ by inhibiting ROS mediated sphingosine kinase 1 in hypoxic PC-3 prostate cancer cells. Bioorg. Med. Chem. Lett., 24, 2560-2564 (2014).

12) Seo DB, Jeong HW, Lee SJ, Lee SJ. Coumestrol induces mitochondrial biogenesis by activating Sirt1 in cultured skeletal muscle cells. J. Agric. Food Chem., 62, 4298-4305 (2014).

13) Chang TS. An updated review of tyrosinase inhibitors. Int. J. Mol. Sci., 10, 2440-2475 (2009). 